\title{
Penetration and efficacy of transdermal NSAIDs in a model of acute joint inflammation
}

This article was published in the following Dove Press journal: Journal of Pain Research

\author{
David Charles Baranowski' \\ Beth Buchanan' \\ Heather C Dwyer' \\ Joseph P Gabriele' \\ Stephanie Kelly ${ }^{2}$ \\ Joseph A Araujo 3 \\ 'Department of Research and \\ Development, Delivra Corp., \\ Charlottetown, PE, Canada; ${ }^{2}$ Vivocore \\ Inc, Toronto, ON, Canada; ${ }^{3}$ nterVivo \\ Solutions Inc, Toronto, ON, Canada
}

\begin{abstract}
Purpose: Prescription and OTC non-steroidal anti-inflammatory drugs (NSAIDs) are ubiquitous treatments for pain and inflammation; however, oral administration of these drugs may produce gastrointestinal (GI) side effects. Transdermal (TD) administration of NSAIDs circumvents these adverse events by avoiding the GI tract and, presumably, achieves regional drug levels of therapeutic effect and thereby, fewer off-target complications.
\end{abstract}

Methods: A drug quantification method was developed for ibuprofen and celecoxib in canine plasma and synovial fluid using liquid chromatography and mass spectrometry. This method was employed to evaluate the penetrance of ibuprofen and celecoxib topical formulations in dogs. Effectiveness of these topical NSAID formulations was compared to the equivalent oral drug concentration in a canine sodium-urate model of acute joint inflammation. In this model, pain was quantified using a modified Canine Brief Pain Inventory questionnaire and regional inflammation using joint caliper measurements; the significance of intervention was evaluated using linear mixed models for repeated measures along with Bonferroni corrections.

Results: After seven days of chronic topical administration, Delivra ${ }^{\mathrm{TM}}$ (DEL) formulations of ibuprofen and celecoxib generated serum levels of $2.9 \mu \mathrm{g} / \mathrm{mL}$ and $220 \mathrm{ng} / \mathrm{mL}$ and synovial fluid levels of $1.8 \mu \mathrm{g} / \mathrm{mL}$ and $203 \mathrm{ng} / \mathrm{mL}$ (respectively). In the canine model of acute inflammation, the overall treatment effects as well as the treatment by time interactions were strongly significant $(P<0.001)$ for both drugs. Oral ibuprofen proved uniquely effective at the earliest time point, while all ibuprofen formulations were effective at treating pain at 8.5 and 24.5 hours post-induction. Similarly, all celecoxib formulations (oral and topical) were equally effective at 8.5 and 24.5 hours post-induction.

Conclusion: DEL formulations of ibuprofen and celecoxib successfully introduced these NSAIDs into synovial fluid at concentrations similar to those observed in circulation. Furthermore, these formulations reduced symptoms of pain associated with acute inflammation. Oral and transdermally delivered NSAIDs have similar pain relief effects; therefore, a replacement or combinatorial treatment may provide a more stable pain relief profile. In conclusion, this work supports further investigation of TD products in the treatment of regional inflammatory events.

Keywords: osteoarthritis, ibuprofen, celecoxib, pain, canine model

\section{Introduction}

Arthritis is the most common musculoskeletal disease, affecting millions of people and pets worldwide. ${ }^{1,2}$ This chronic disease is characterized by symptoms of cartilage loss, synovium inflammation, osteophytosis, reduced range of motion, and pain, which eventually result in joint failure. ${ }^{3}$ The etiology of arthritis is multifactorial and linked to inflammation, trauma, aging, obesity, chondrocyte differentiation, and genetic pre-
Baranowsk

Delivra Corp., NRC-PEI, Suite 407, 550

University Ave, Charlottetown, PE, CIA

4P3, Canada

Tel + I 9023673225

Email dbaranowski@delivrainc.com 
disposition. ${ }^{3}$ Therapeutics for disease reversal are limited, and consequently the pharmaceutical industry continues to focus on improvement of three critical and currently druggable target endpoints: pain, function, and disease progression. ${ }^{3}$ Pain and disability are the most common complaints of arthritis patients, ${ }^{4}$ symptoms that are primarily managed with NSAIDs ${ }^{5}$

Classic NSAIDs like ibuprofen $((R, S)$-2-(4-(2-methylpropyl)phenyl)propanoic acid) have analgesic, anti-inflammatory and antipyretic effects due to inhibition of prostaglandin synthesis by non-specific inhibition of both COX-1 and COX2enzymes. ${ }^{6}$ Inhibition of COX-1 is hypothesized to cause GI side effects, ${ }^{7,8}$ which occur in approximately $15 \%$ of patients and include (but are not limited to) nausea, diarrhea, and constipation. Gastric upset and ulceration are even more pronounced in dogs and cats; therefore, ibuprofen use is discouraged in companion animals., ${ }^{9,10}$ GI adverse events associated with oral ibuprofen use make topical formulation a promising alternative for both human and veterinary medicine. ${ }^{11,12} \mathrm{By}$ contrast, celecoxib (4-[5-(4-Methylphenyl)-3(trifluoromethyl) pyrazol-1-yl] benzenesulfonamide) is a COX-2 specific inhibitor that was originally patented by Pfizer under the brand names Celebrex and Celebra and is currently available as a generic under many other brands. Like ibuprofen, celecoxib is used to treat pain and fever and has established clinical efficacy for osteoarthritis, rheumatoid arthritis, and acute postoperative pain. ${ }^{13-17}$ Unlike ibuprofen, celecoxib has reduced instances of GI side effects, hypothetically due to its limited COX-1 inhibitory activity. ${ }^{18,19}$ Celecoxib is also linked to increased incidence of major vascular events, including myocardial infarction and stroke, and is, therefore, unlikely to become an OTC drug like ibuprofen. ${ }^{20}$ In summary, NSAID use may result in off-target adverse effects on the GI and cardiovascular systems; therefore, administration via a route that avoids or reduces systemic drug exposure, while achieving targeted pain reduction, is desirable. TD NSAID products may embody these advantageous criteria and have been investigated in a variety of clinical trials with some (eg, Voltaren $^{\circledR}$ [diclofenac]) commercial success. ${ }^{11,12,21-23}$

The development of novel treatments and formulations for joint inflammation requires evaluation in relevant animal models. ${ }^{3}$ Large animals, such as dogs, are used to study disease processes and treatment because of the similarity of their anatomy with that of humans ${ }^{13}$ and because models in rodents do not accurately predict the incidence and severity of arthritis related inflammation, ${ }^{24}$ nor changes in gait due to experiential pain. ${ }^{4}$ Canine models of joint inflammation attempt to mirror the most common human diagnoses which are osteoarthritic, rheumatic, or gout and each have an estimated prevalence of $19 \%, 1 \%$, and $1.5 \%$ in humans..$^{25,26}$ While the etiology of osteoarthritis is largely idiopathic, rheumatoid arthritis and gout are unequivocally linked to the immune system, with the latter disease yielding an inflammatory response to sodium urate crystals. ${ }^{27-29}$ The injection of sodium urate within the joint space causes a similar inflammatory response and is established as a model of joint pain in beagle dogs for the evaluation of novel analgesics. ${ }^{13,30}$

In the current study, DEL topical formulations of ibuprofen and celecoxib were evaluated for their ability to introduce the active drug into the synovial fluid of dogs. The analgesic and anti-inflammatory effect of these test articles were also evaluated in a sodium urate-induced model of acute joint inflammation.

\section{Materials and methods Laboratory sample analysis}

A liquid chromatography tandem mass spectrometry (LCMS/MS) method, operated in multiple-reaction monitoring mode, was optimized using ibuprofen and celecoxib standards. The analysis also included a solid-phase extraction method to extract ibuprofen and celecoxib from serum and synovial fluid matrices. Optimized methods were used to quantify ibuprofen and celecoxib in biological samples to support penetration studies.

\section{Chemicals, reagents, and instrumentation}

Celecoxib (C251000) and rac-d $\mathrm{d}_{3}$-ibuprofen (I140002) were purchased from Toronto Research Chemicals, Toronto, ON, Canada. $\mathrm{d}_{7}$-celecoxib (D-7317) was purchased from CDN Isotopes, Pointe-Claire, QC, Canada. Rac-ibuprofen (I4833), ammonium acetate (A7330), and phosphoric acid (438081) were purchased from Sigma Aldrich, St. Louis, MO, United States. Methanol (6701-7-40), acetonitrile (1401-7-40) and ammonium hydroxide (1525-1) were acquired from Caledon Laboratories, Georgetown, ON, Canada. De-ionized water was collected from a Millipore A10 Advantage system, Etobicoke, ON, Canada. Formic acid (FX0440-5) was purchased from EMD Millipore, Etobicoke, ON, Canada. DEL-SR formulation base (13750-2) was provided by Delivra Corp., Hamilton, ON, Canada.

Solid phase extraction was performed on Oasis MAX $\mu$ Elution Plates $30 \mu \mathrm{m}$ (186001829), Oasis HLB $\mu$ Elution Plates $30 \mu \mathrm{m}$ (186001828BA), Oasis MAX $1 \mathrm{cc}$ (30 mg) Extraction Cartridge (186000366) and Oasis HLB 1 cc (30 $\mathrm{mg}$ ) extraction cartridge (WAT094225) provided by Waters Corporation, Milford, MA, USA. A 4000 QTRAP from AB Sciex Instruments, Concord, ON, Canada, equipped with an 
1100 HPLC from Agilent Technologies, Mississauga, ON, Canada, was used for the analysis.

\section{Chromatographic conditions}

Gradient chromatographic elution of ibuprofen and celecoxib were performed on a HALO C18 HPLC column $(2.1 \times 100$ mm x $2.7 \mu \mathrm{m}$ - Advanced Materials Technology Inc., Wilmington, DE, USA). The ibuprofen solvent program was initiated at 70:30 (5 mM ammonium acetate in water [A]: and 5 $\mathrm{mM}$ ammonium acetate in 5:95 water:acetonitrile [B]: and raised to $100 \% \mathrm{~B}$ linearly in three minutes, held at $100 \%$ $\mathrm{B}$ for 1 minute and reverted to starting conditions by 4.5 minutes. The column was then allowed to re-equilibrate for 5 minutes. The injection volume was $5 \mu \mathrm{L}$, the column was maintained at $25^{\circ} \mathrm{C}$ and the flow rate was constant at 400 $\mu \mathrm{L} / \mathrm{min}$. The HPLC gradient conditions for celecoxib began with 50: $50(\mathrm{~A}: \mathrm{B})$ and were increased to $100 \% \mathrm{~B}$ over the first three minutes, held at $100 \% \mathrm{~B}$ for 1 minute, reverted to 50:50 A:B by 4.5 minutes and re-equilibrated for 5 minutes.

\section{Mass spectrometer conditions}

The mass spectrometer was operated in negative ionization mode for quantification of ibuprofen and $\mathrm{d}_{3}$-ibuprofen. The curtain gas was $15 \mathrm{psi}$, electrospray voltage was $-4500 \mathrm{~V}$, gas sources 1 and 2 were 50 psi, the heater interface was $100^{\circ} \mathrm{C}, \mathrm{CAD}$ gas was high, the declustering potential was $-60 \mathrm{~V}$; entrance potential $-10 \mathrm{~V}$, the collision energy was $-10 \mathrm{~V}$, and collision exit potential was $-12 \mathrm{~V}$. Quantification was performed using the MRM transitions $205 \rightarrow 161$ for ibuprofen, and $208 \rightarrow 164$ for $\mathrm{d}_{3}$-ibuprofen, both having a dwell time of $100 \mathrm{msec}$.

Quantification of celecoxib and $\mathrm{d}_{7}$-celecoxib was performed in negative ionization mode. The curtain gas was 15 psi, the electrospray voltage was $-4500 \mathrm{~V}$, gas sources 1 and 2 were $50 \mathrm{psi}$, the heater interface was $100^{\circ} \mathrm{C}$, the CAD gas was high, the declustering potential was $-70 \mathrm{~V}$, the entrance potential $-10 \mathrm{~V}$, the collision energy was $-35 \mathrm{~V}$, and the collision exit potential was $-10 \mathrm{~V}$. Quantification was performed using the MRM transitions $380 \rightarrow 316$ for celecoxib, and 387 $\rightarrow 323$ for $\mathrm{d}_{7}$-celecoxib both having a dwell time of $100 \mathrm{msec}$.

\section{Standard solutions, calibration curves, and quality control samples}

Individual $5 \mathrm{mg} / \mathrm{mL}$ stock solutions of ibuprofen, celecoxib, $\mathrm{d}_{3}$-ibuprofen, and $\mathrm{d}_{7}$-celecoxib were prepared in methanol. Stock solutions were further diluted with $1: 1$ methanol:acetonitrile to prepare a dilution with the following concentrations of ibuprofen or celecoxib: 1, 5, 10, 50, 100,
$500,1000,5000$, and 10,000 ng/mL along with a constant IS)concentration of $250 \mathrm{ng} / \mathrm{mL}$. Ratio of analyte peak area to IS peak area was plotted against concentration to give a calibration curve that was fit with a linear regression equation of the form $y=m x+b$.

\section{Biological sample preparation}

For the preparation of samples for ibuprofen quantification, a MAX $\mu$ Elution plate was conditioned with methanol (200 $\mu \mathrm{L})$ and then equilibrated with $\mathrm{H}_{2} \mathrm{O}(200 \mu \mathrm{L})$. Biofluid matrix (serum or synovial fluid: $50 \mu \mathrm{L}$ ) was thawed and mixed with $\mathrm{H}_{2} \mathrm{O}(200 \mu \mathrm{L})$ and $4 \% \mathrm{H}_{3} \mathrm{PO}_{4}(250 \mu \mathrm{L})$. The mixture was loaded onto the plate and then passed drop wise through the sorbent under vacuum. The solid phase was washed with $5 \% \mathrm{NH}_{4} \mathrm{OH}(200 \mu \mathrm{L})$ and then with $5 \%$ methanol $(2 \times 25$ $\mu \mathrm{L})$. The analytes were then eluted into a clean 96 well plate with $2 \%$ formic acid in acetonitrile $(3 \times 25 \mu \mathrm{L})$. The eluent was combined with $75 \mu \mathrm{L}$ of $\mathrm{d}_{3}$-ibuprofen $(500 \mathrm{ng} / \mathrm{mL}$ in methanol) mixed and injected for analysis. Quality control samples were prepared from blank matrix at concentrations of 100,250 , or $500 \mathrm{ng} / \mathrm{mL}$.

For the preparation of samples for celecoxib quantification, an HLB $\mu$ Elution plate was conditioned with methanol $(200 \mu \mathrm{L})$ and then equilibrated with $\mathrm{H}_{2} \mathrm{O}(200 \mu \mathrm{L})$. Biofluid matrix (serum or synovial fluid $50 \mu \mathrm{L}$ ) was thawed and mixed with $\mathrm{H}_{2} \mathrm{O}(200 \mu \mathrm{L})$ and $4 \% \mathrm{H}_{3} \mathrm{PO}_{4}(250 \mu \mathrm{L})$, loaded onto the plate, and eluted drop wise under vacuum. The solid phase was washed with $\mathrm{H}_{2} \mathrm{O}(200 \mu \mathrm{L})$ followed by $5 \%$ methanol $(2 \times 25 \mu \mathrm{L})$, and then the analytes were eluted into a clean 96 well plate with acetonitrile $(3 \times 25 \mu \mathrm{L})$. The eluent was combined with $75 \mu \mathrm{L}$ of $\mathrm{d}_{7}$-celecoxib (500 $\mathrm{ng} / \mathrm{mL}$ in methanol), mixed, and injected for analysis. Quality control samples were prepared from blank matrix at concentrations of 100 , 250 , or $500 \mathrm{ng} / \mathrm{mL}$.

\section{Formulation construction $4 \%$ ibuprofen in DEL (16-03|-0|8)}

A topical formulation of ibuprofen was prepared by first dissolving the drug $(200 \mathrm{mg})$ in Transcutol CG $(0.2 \mathrm{~mL})$. DEL-SR (4.6 g) was then added to the solution, and the mixture was homogenized using vortexing, sonication, and manual stirring. The $\mathrm{pH}$ of the formulation was 4.0-5.0. The formulation was stored in an airtight, light-protected container until tested.

\section{$8 \%$ celecoxib in DEL (15-640-253)}

A topical formulation of celecoxib was prepared by first dissolving the drug $(400 \mathrm{mg})$ in Transcutol CG $(0.8 \mathrm{~mL})$. 
DEL-SR (3.8 g) was then added to the solution, and the mixture was homogenized using vortexing, sonication, and manual stirring. The $\mathrm{pH}$ of the formulation was 4.5-5.5. The formulation was stored in an airtight, light-protected container until tested.

\section{$4 \%$ ibuprofen-PLO (16-039-020)}

A topical formulation of ibuprofen was prepared by first dissolving the drug $(200 \mathrm{mg})$ in Transcutol CG $(0.2 \mathrm{~mL})$. PLO (4.6 g) was then added to the solution, and the mixture was homogenized using vortexing, sonication, and manual stirring. The $\mathrm{pH}$ of the formulation was 4.0-5.0. The formulation was stored in an airtight, light-protected container until tested.

\section{8\% celecoxib-PLO (16-037-020)}

A topical formulation of celecoxib was prepared by first dissolving the drug (400 mg) in Transcutol CG $(0.8 \mathrm{~mL})$. PLO ( $3.8 \mathrm{~g}$ ) was then added to the solution, and the mixture was homogenized using vortexing, sonication, and manual stirring. The $\mathrm{pH}$ of the formulation was 4.5-5.5. The formulation was stored in an airtight, light-protected container until tested.

\section{Animal studies}

The animal protocols for the studies were conducted by a contract research organization (InterVivo Solutions, Toronto, ON, Canada) and approved (approval number 2015Generic10-VRI58-16004-CE-2) by the facility's ACC in accordance with the principles of the Animal for Research Act of Ontario and the guidelines of Canadian Council on Animal Care CCAC. All animals survived the study and were returned to the InterVivo canine colony at study conclusion.

\section{Topical NSAID penetration assessment}

Seventeen aged beagle dogs ( $>6$ years) of both sexes with quantified radiographic evidence of bilateral shoulder osteoarthritis were used for the study. Six dogs were treated topically with 4\% ibuprofen formulated in DEL-SR ( $1 \mathrm{~g}$ cream per affected joint twice daily; $160 \mathrm{mg}$ ibuprofen total/day), six were treated topically with $3 \%$ celecoxib in DEL ( $1 \mathrm{~g}$ cream per affected joint twice daily; $120 \mathrm{mg}$ total celecoxib/ day) and five were treated with the DEL-SR ( $1 \mathrm{~g}$ cream per affected joint twice daily), which served as the negative vehicle control. The treatment cycle consisted of seven days of treatment, followed by a seven-day washout period devoid of treatment. Blood and synovial fluid samples were collected 60 minutes post treatment on the seventh day of drug application and on the seventh day of the washout period.
Synovial fluid was collected under sedation (medetomidine [0.01 mg/kg] and butorphanol [0.1 mg/kg]) by IV. The fluid was collected from stifle or shoulder joints by arthrocentesis following aseptic surgical preparation of the area.

\section{NSAID efficacy assessment using sodium urate induced joint synovitis}

A randomized, blind, controlled, preclinical study was employed to evaluate various administrations of ibuprofen and celecoxib in a sodium urate model in beagle dogs. Prior to drug administration and sodium urate injection, the stifle joints were shaved and aseptically cleaned. TD treatment doses were measured in milligrams using an analytical scale and then applied directly to the induced stifle joint. TD treatments were rubbed into the dog's skin for a minimum of 1 minute. Oral treatment doses were measured in milligrams using an analytical scale and contained within gelatin capsules. Administration of the capsule was conducted with attention to complete delivery and retention of the entire intended dose. Oral ibuprofen ( $4 \mathrm{mg} / \mathrm{kg}), 4 \%$ ibuprofen in PLO (100 mg/kg), and 4\% ibuprofen in DEL-SR (100 mg/kg) were administered in equimolar dosages. Likewise, oral celecoxib (3 mg/kg), 8\% celecoxib in PLO (37.5 mg/kg), and 8\% celecoxib in DEL-SR $(37.5 \mathrm{mg} / \mathrm{kg})$ were also administered in equimolar dosages. Negative controls included untreated animals and vehicle treatment (DEL-SR; $100 \mathrm{mg} / \mathrm{kg}$ ). Each treatment group consisted of eight animals with average dispersion of age $(6.3 \pm 2.8$ years), weight $(10.7 \mathrm{~kg} \pm 1.5)$, and sex $(1: 1.13$; female:male) that were in good general health as determined by historical health records. Subjects had no clinical or radiographic signs of osteoarthritis and a baseline cumulative pain score of 5 or less, as determined using the previously validated CBPI questionnaire. Animals were observed twice daily over the course of the study for adverse events. The body weight of each dog was measured and used to determine individual treatment doses of sodium urate and orally administered drugs.

\section{Anesthesia and sodium urate injection}

Joint induction of synovitis by sodium urate injection involved the following procedures. Anesthesia was induced with propofol ( $8 \mathrm{mg} / \mathrm{kg}$ IV to effect). Then subjects were intubated and anesthesia was maintained with an isofluraneoxygen mixture. The induction injectate was prepared with sodium urate crystals that were mixed with sterile saline to produce a solution with a concentration of $20 \mathrm{mg} / \mathrm{mL}$. The solution was sonicated for 60 minutes and the $\mathrm{pH}$ was adjusted, with the addition of either hydrochloric acid or 
sodium hydroxide, to a suitable level (between 6.9 and 7.2) for injection. Following aseptic surgical preparation of the injection area, the joint was aspirated. The presence of synovial fluid confirmed an accurate location for the injection. For each animal, $1 \mathrm{~mL}$ of the sodium urate solution was injected randomly into the stifle, either the right or the left.

\section{Administration of test articles}

Test and control article administration occurred 30 minutes following synovitis induction with the sodium urate injection. TD treatment doses were measured in milligrams using an analytical scale and were then applied directly to the induced stifle. These preparations were rubbed into the dog's skin for a minimum of 1 minute. Oral treatments were administered with attention to complete delivery and retention of the entire intended dose.

\section{Pain questionnaire assessment}

The pain questionnaire is a laboratory adaption of the validated CBPI questionnaire, which is a clinical questionnaire used to evaluate pain level based on owner's responses. Modifications to the questionnaire account for differences between owner pain evaluation of pets and pain evaluation of laboratory dogs by technical staff. Specifically, the ability of each dog to rise to standing from lying down, walk, run and climb stairs, curbs, and doorsteps was replaced with their ability to walk, trot, gallop, rear, jump over a low obstacle, climb and descend stairs, and jump down from a perch. The evaluation of general activity, with parallel subjective evaluation of pain observed during each behavior remained unchanged. Assessments were performed by the same-trained technician across the study. All subjects were screened with the pain questionnaire prior to the sodium urate injection. On the days that sodium urate was injected, the animals were assessed with the questionnaire at 4.5, 8.5, 24.5, and 30.5 hours ( \pm 15 minutes) following induction of synovitis. At each of these time points, the pain questionnaire was administered immediately following measurement of the patella/patellar tendon (knee cap) width as described below.

\section{Caliper measurements}

Within thirty minutes of synovitis induction and 4.5, 8.5, 24.5 , and 30.5 hours ( \pm 15 minutes) following induction, the width of the patella and the patellar tendon were measured in millimeters using Venier $150 \mathrm{~mm}$ plastic gage calipers which measured in increments of $1 \mathrm{~mm}$. Measurements of both the left and right stifle were performed by the same technician, who took three separate caliper measurements of each joint.
The mean of the three measurements from each joint was used for analysis.

\section{Statistical analysis}

Graphpad Prism 6 software was used for all data analysis and figure generation. For the comparison of drug penetration data (i.e., treated vs washout), paired $t$-tests were used to evaluate the significance. For the analysis of treatment efficacy in the sodium urate model, linear mixed models for repeated measures on subjects over time were fitted with Stata version 15 software. The analysis included responses at $4.5,8.5$, and 24.5 hours where pain scores were above negligible levels. Linear contrasts were used to assess the comparisons of primary interest between effects of each drug, averaged across its three formulations, compared to control (average of no intervention and vehicle groups), with a Bonferroni adjustment of the significance level for testing at 3 time points. Additionally, pairwise comparisons were performed among the formulations at these 3 time points, also with Bonferroni adjustments for multiple testing within each drug.

\section{Results \\ Optimization of LCMS conditions and sample preparation}

HPLC conditions were optimized using reversed-phase separation and MS parameters for each analyte separately to obtain optimal sensitivity. Deuterated IS: $d_{3}$-ibuprofen $\mathrm{d}_{7}$-celecoxib were used in the analysis. After a series of optimization experiments, it was found that ibuprofen was best extracted using anion exchange sorbent and celecoxib using a reversed phase sorbent, both methods achieving recovery greater than $79 \%$.

\section{Calibration curves, LLOQ and extraction recovery}

The calibration curves for ibuprofen and celecoxib were linear from 5 to $10,000 \mathrm{ng} / \mathrm{mL}$ (ibuprofen: $\mathrm{y}=0.0047 \mathrm{x}+0.0064$, $\mathrm{R}^{2}=0.9996$, celecoxib: $\mathrm{y}=0.0025 \mathrm{x}+0.0725, \mathrm{R}^{2}=0.9998$ ). The LLOQ was $2.5 \mathrm{ng} / \mathrm{mL}$ for both analytes. Extraction of analytes from biological matrices was optimized using SPE. Extraction recovery was evaluated at three concentrations with optimized methodology for each analyte calculated using the equation below. The results are summarized in Table 1.

Extraction recovery $=\frac{\text { ratio } \frac{\text { analyte }}{\mathrm{Is}} \text { peak area of matrix spiked with analyte }}{\text { ratio } \frac{\text { analyte }}{\mathrm{Is}} \text { peak area of matrix extract spiked with analyte }}$ 


\section{Serum and synovial levels of NSAIDs following topical administration}

Average serum concentrations of ibuprofen after 7 days of topical administration were $2.9 \pm 0.5 \mu \mathrm{g} / \mathrm{mL}$ (mean \pm SEM) (Figure 1). Similarly, the average ibuprofen level in synovial fluid was $1.8 \pm 0.3 \mu \mathrm{g} / \mathrm{mL}$ (mean \pm SEM) after the treatment period. Following a washout period of seven days without treatment, drug levels were significantly decreased, $\sim 40$-fold, in serum and synovial fluid to $67.6 \pm 13.2$ and $37.8 \pm 10.0 \mathrm{ng} /$ $\mathrm{mL}$, respectively (Figure 1).

Average serum concentration of celecoxib after 7 days of topical administration was $220.9 \pm 92.7 \mathrm{ng} / \mathrm{mL}$ (mean \pm SEM)
(Figure 2). Similarly, the average celecoxib level in synovial fluid was $203.0 \pm 0.3 \mathrm{ng} / \mathrm{mL}$ (mean \pm SEM) after the treatment period. Following a washout period of seven days without treatment, drug levels decreased in serum and synovial fluid to $4.2 \pm 2.2$ and $16.8 \pm 15.5 \mathrm{ng} / \mathrm{mL}$, respectively (Figure 2), however this decrease was not significant.

\section{The efficacy of topical NSAIDs in acute joint inflammation}

The efficacy of each intervention was evaluated by measuring changes in swelling using caliper diameter measurements and CBPI pain scores at baseline and at 4.5, 8.5, 24.5, and 30.5

Table I Extraction recoveries of ibuprofen and celecoxib from serum and synovial fluid

\begin{tabular}{|l|l|l|l|}
\hline Analyte & $\begin{array}{l}\text { Concentration } \\
(\mathbf{n g} / \mathbf{m L})\end{array}$ & $\begin{array}{l}\text { Extraction recovery from } \\
\text { serum (\%) (mean } \pm \text { SEM) }\end{array}$ & $\begin{array}{l}\text { Extraction recovery from } \\
\text { synovial fluid (\%) (mean } \pm \text { SEM) }\end{array}$ \\
\hline \multirow{4}{*}{ Ibuprofen } & 100 & $91 \pm 6$ & $139 *$ \\
\cline { 2 - 4 } & 250 & $92 \pm 7$ & $113 \pm 5$ \\
\cline { 2 - 4 } & 500 & $79 \pm 7$ & $128^{*}$ \\
\hline \multirow{5}{*}{ Celecoxib } & 100 & $98 \pm 4$ & - \\
\cline { 2 - 4 } & 250 & $90 \pm 3$ & $104 \pm 4$ \\
\cline { 2 - 4 } & 500 & $101 \pm 4$ & $81^{*}$ \\
\hline
\end{tabular}

Notes: Extraction recovery values from serum and synovial fluid are expressed as \% nominal value along with the standard error of the mean (SEM). *Denotes a single replicate due to limited sample matrix.
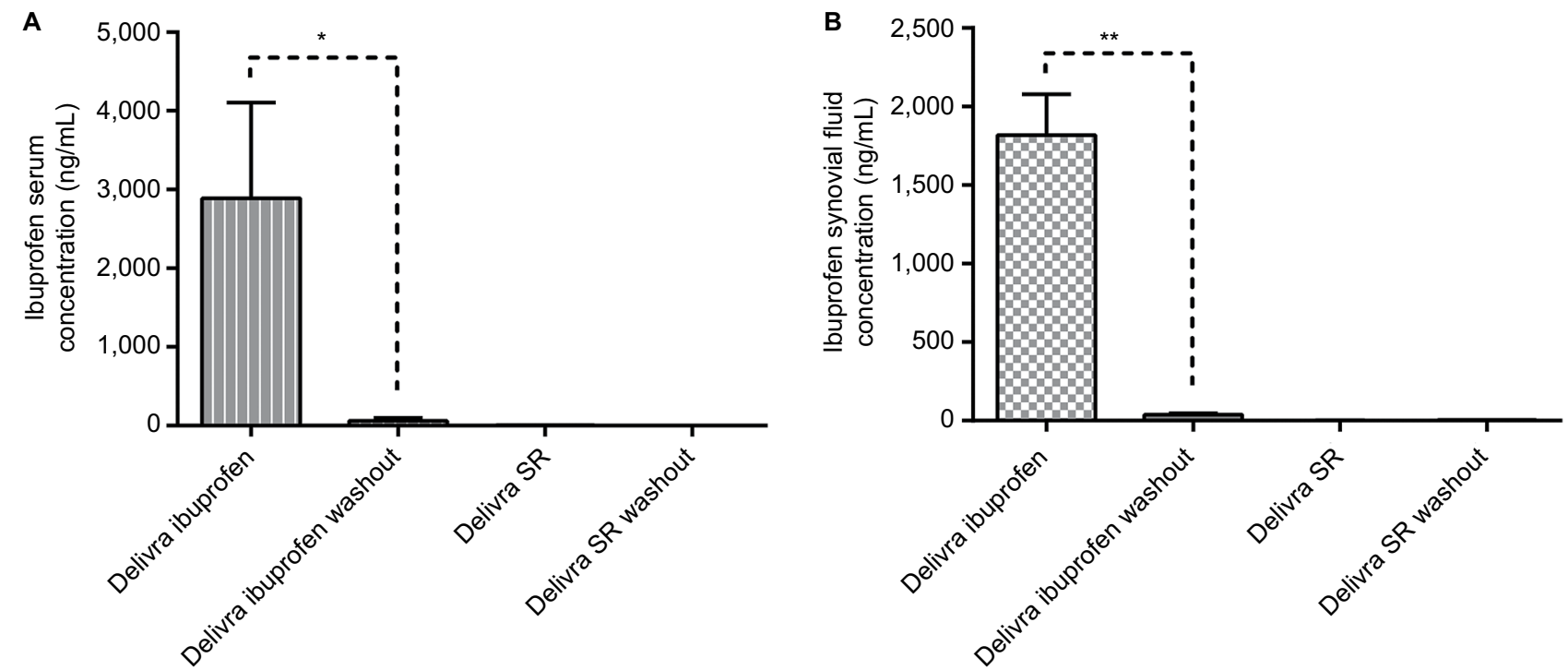

Figure I Serum and synovial fluid ibuprofen concentration after chronic topical administration and washout.

Notes: Aged beagles received I g cream per shoulder joint twice/day, either $4 \%$ ibuprofen formulated in Delivra ${ }^{\mathrm{TM}}$ SR or vehicle only (Delivra ${ }^{\mathrm{TM}}$ SR) for 7 days. Serum (A) and synovial fluid (B) were collected 60 minutes post final administration. The same bio-fluids were sampled again after a seven-day washout period. Ibuprofen concentrations measured by LC-MS/MS were 2,910 $\pm 544.2 \mathrm{ng} / \mathrm{mL}$ (mean \pm SEM), $67.6 \pm 13.2 \mathrm{ng} / \mathrm{mL}$ (mean \pm SEM), $5.3 \pm 2.1 \mathrm{ng} / \mathrm{mL}$ (mean \pm SEM) for BLQ in serum (A) for Delivra ${ }^{\text {TM }}$-ibuprofen $(n=6)$, Delivra ${ }^{T M}$-ibuprofen washout, Delivra ${ }^{T M} S R(n=2)$ and Delivra ${ }^{T M} S R$ washout, respectively. Ibuprofen concentration in synovial fluid $(B)$ was $I, 8 I 7 \pm 262.0 \mathrm{ng} / \mathrm{mL}(\mathrm{mean}$ $\pm S E M$ ), $37.8 \pm 10.0 \mathrm{ng} / \mathrm{mL}$ (mean $\pm S E M$ ), BLQ, and $4.9 \pm 0.1 \mathrm{ng} / \mathrm{mL}$ (mean $\pm S E M$ ) for Delivra ${ }^{T M}$-ibuprofen, Delivra ${ }^{T M}$-ibuprofen washout, Delivra ${ }^{T M} S$ SR and Delivra ${ }^{T M}$ SR washout, respectively. Paired $t$-tests demonstrated a significant reduction in ibuprofen levels between chronic treatment and washout periods for $(\mathbf{A})$ serum $(* P=0.008)$ and $(\mathbf{B})$ synovial fluid (**P=0.004).

Abbreviations: DEL, Delivra ${ }^{\mathrm{TM}}$; DEL-SR, Delivra ${ }^{\mathrm{TM}}$ slow release; SEM, standard error of the mean; BLQ, below the limit of quantification; LC-MS/MS, liquid chromatography tandem mass spectrometry. 

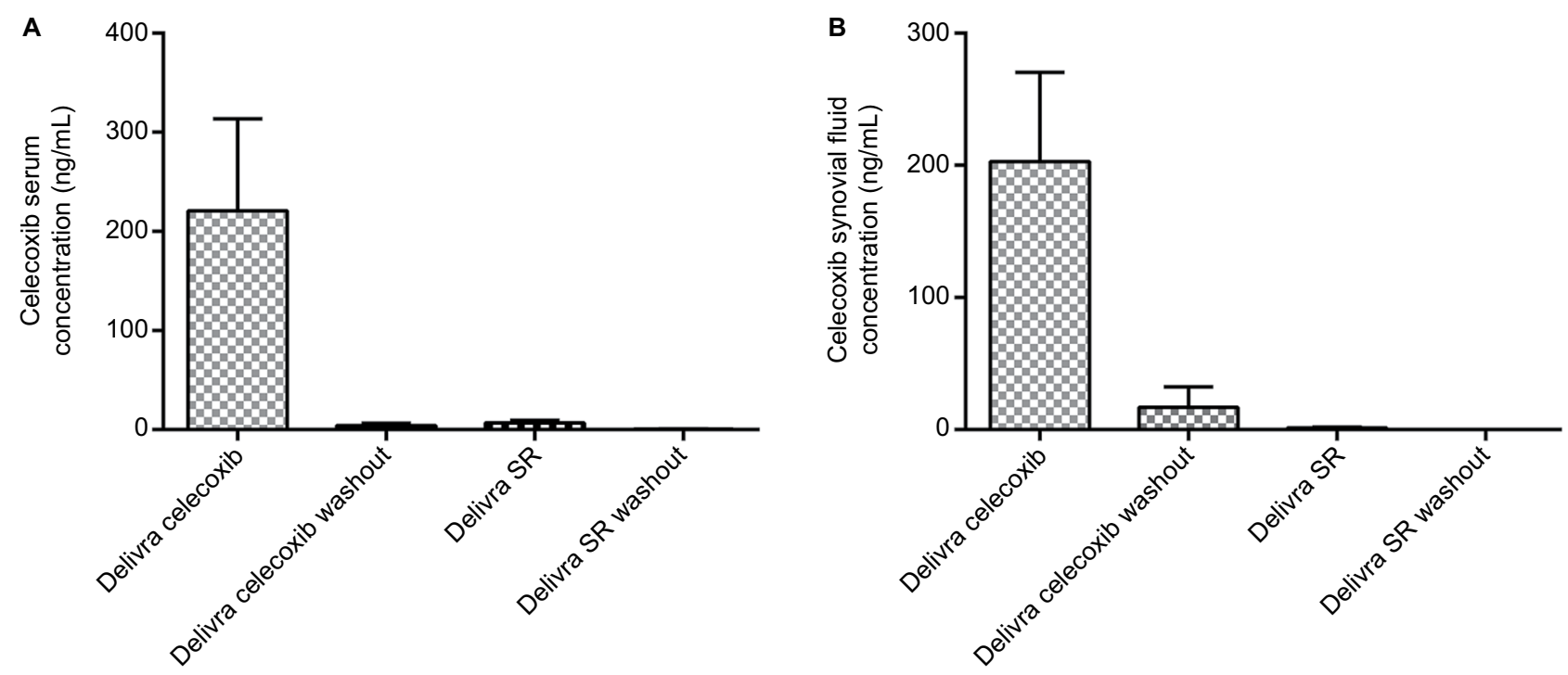

Figure 2 Serum and synovial fluid celecoxib concentrations after chronic topical administration and washout.

Notes: Aged beagles received I g cream per shoulder joint twice/day, either $3 \%$ celecoxib formulated in Delivra ${ }^{T M}$ SR (Delivra ${ }^{T M}$ celecoxib) or vehicle (Delivra ${ }^{T M}$ SR) for 7 days. Serum (A) and synovial fluid (B) were collected 60 minutes post final administration. The same bio-fluids were sampled again after a seven-day washout period. Celecoxib concentrations were measured by LC-MS/MS and were $220.9 \pm 92.7 \mathrm{ng} / \mathrm{mL}$ (mean \pm SEM), $4.2 \pm 2.2 \mathrm{ng} / \mathrm{mL}$ (mean \pm SEM), $7.0 \pm 2.5 \mathrm{ng} / \mathrm{mL}$ (mean \pm SEM), below the limit of quantification (BLQ) in (A) serum for Delivra ${ }^{T M}$-celecoxib $(n=6)$, Delivra ${ }^{T M}$-celecoxib washout, Delivra SR ( $\left.n=3\right)$ and Delivra ${ }^{T M} S R$ washout, respectively. Celecoxib concentration in (B) synovial fluid was $203.0 \pm 67.3 \mathrm{ng} / \mathrm{mL}$ (mean $\pm S E M$ ), $16.8 \pm 15.5 \mathrm{ng} / \mathrm{mL}$ (mean $\pm S E M$ ), BLQ and BLQ for Delivra ${ }^{T M}$-celecoxib, Delivra ${ }^{T M}$-celecoxib washout, Delivra $^{T M} S R$ and Delivra ${ }^{T M} S R$ washout, respectively. Paired $t$-tests demonstrated that the reduction in celecoxib levels between chronic treatment and washout periods for (A) serum $(P=0.07)$ and $(B)$ synovial fluid $(P=0.09)$ were not significant.

Abbreviations: DEL, Delivra ${ }^{T M}$; DEL-SR, Delivra ${ }^{\text {TM }}$ slow release; LC-MS/MS, liquid chromatography tandem mass; BLQ, below the limit of quantification; SEM, standard error of the mean.

hours after synovitis induction. For the analysis of treatment efficacy in the sodium urate model, a linear mixed model for repeated measures on subjects over time was employed and analysis included responses at 4.5, 8.5, and 24.5 hours where pain scores were above negligible levels. A significance level of $P<0.05$ was used in the analysis along with a Bonferroni adjustment for multiple comparisons. Oral ibuprofen was the only treatment that moderated pain at the earliest time point, demonstrating a significant improvement in observed pain compared to no treatment at 4.5 hours (Figure 3A). For further time points, the contrasts comparing overall drug effects to control at 8.5 and 24.5 hours were all statistically significant and no significant differences were found between drug formulations (Figure 3A). Overall, oral ibuprofen yielded significant pain reduction at the earliest time point while all ibuprofen formulations proved effective and statistically indistinguishable at 8.5 and 24.5 hours (Figure $3 \mathrm{~A})$ In contrast to CBPI scores, caliper measurements failed to show a significant reduction in edema. Results from the ibuprofen-PLO and DEL-ibuprofen groups suggested that the test articles had an anti-nociceptive effect. However, none of the intervention groups observed demonstrated a significant improvement in synovitis related edema (Figure 3B).
Unlike oral ibuprofen, oral celecoxib did not yield a significant reduction in CBPI score in the first 4.5 hours. Regardless of route or formulation, celecoxib proved ineffective for early ( 4.5 hour) pain reduction whereas all three interventions were significant at 8.5 and 24.5 hours and all three celecoxib formulations proved effective and statistically indistinguishable at these time points (Figure 4A). With respect to joint width and swelling, only oral celecoxib suggested a trend of symptom reduction, albeit this intervention (like all others) failed to yield a statistical effect (Figure 4B).

\section{Discussion}

NSAIDs are almost universally administered by oral capsule or solution in both human and veterinary applications. Likewise, in both human and veterinary systems, NSAIDs can produce GI upset, which begs the development of products with alternative routes of administration. ${ }^{7,8}$ In the context of joint inflammation, particularly gout, delivery of the NSAIDs to the site of pain is paramount, which is represented in this study by the measurement and comparison of the synovial fluid and circulating concentrations of the drug.

The present study compared the distribution of ibuprofen in beagle dogs after chronic topical administration $(14.5 \mathrm{mg}$ / 

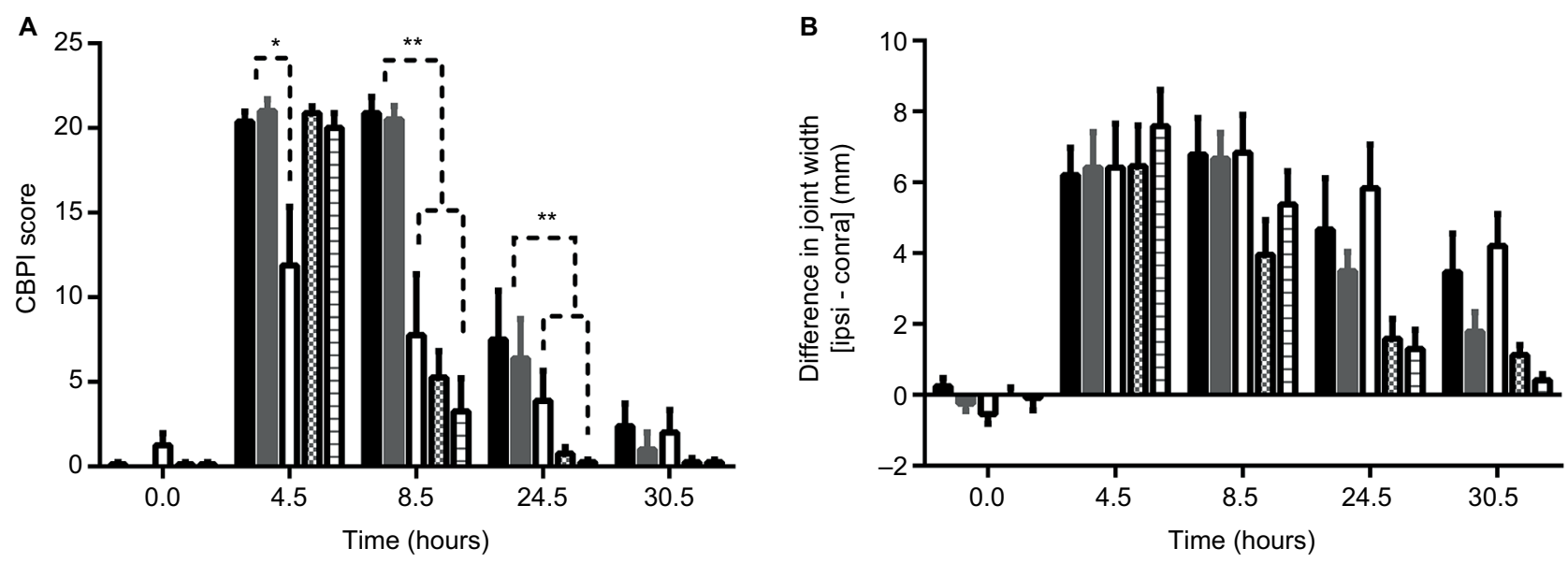

Figure 3 A comparison of oral and TD ibuprofen to alter pain and swelling in a model of acute joint synovitis.

Notes: A study design involving beagle dogs ( $\mathrm{N}=8$ per group) involved baseline $(0.0)$ and post-synovitis measures at $4.5,8.5,24.5$, and 30.5 hours for (A) pain levels using the CBPI and (B) swelling using stifle width $(\mathrm{mm})$ for the ipsilateral and contralateral joints. Inflammatory synovitis was induced by injection of $\mathrm{I} .0 \mathrm{~mL}$ of $20 \mathrm{mg} / \mathrm{mL}$ sodium urate (under anesthesia). Animals received no intervention ( $\mathbf{\square})$, vehicle Delivra ${ }^{\mathrm{TM}} \mathrm{SR}$ ( $100 \mathrm{mg} / \mathrm{kg}$; ), or equimolar interventions consisting of oral ibuprofen (4 mg/kg; ), PLOibuprofen ( $4 \%$ at $100 \mathrm{mg} / \mathrm{kg}$; ), or Delivra ${ }^{\mathrm{TM}}$-ibuprofen $(4 \%$ at $100 \mathrm{mg} / \mathrm{kg})$. Results are graphically represented as the average CBPI score, average joint width along with SEM error bars for each group. Linear mixed models for repeated measures and Bonferroni adjustment for multiple comparisons demonstrated significant pain reduction (A) for oral ibuprofen $(* P<0.00 I)$ at 4.5 hours with all interventions yielding significant $(* * P<0.02)$ and indistinguishable effects at 8.5 and 24.5 hours.

Abbreviations: PLO, pluronic lecithin organogel; DEL, Delivra ${ }^{T M}$; DEL-SR, Delivra ${ }^{T M}$ slow release; CBPI, canine brief pain inventory; TD, transdermal.
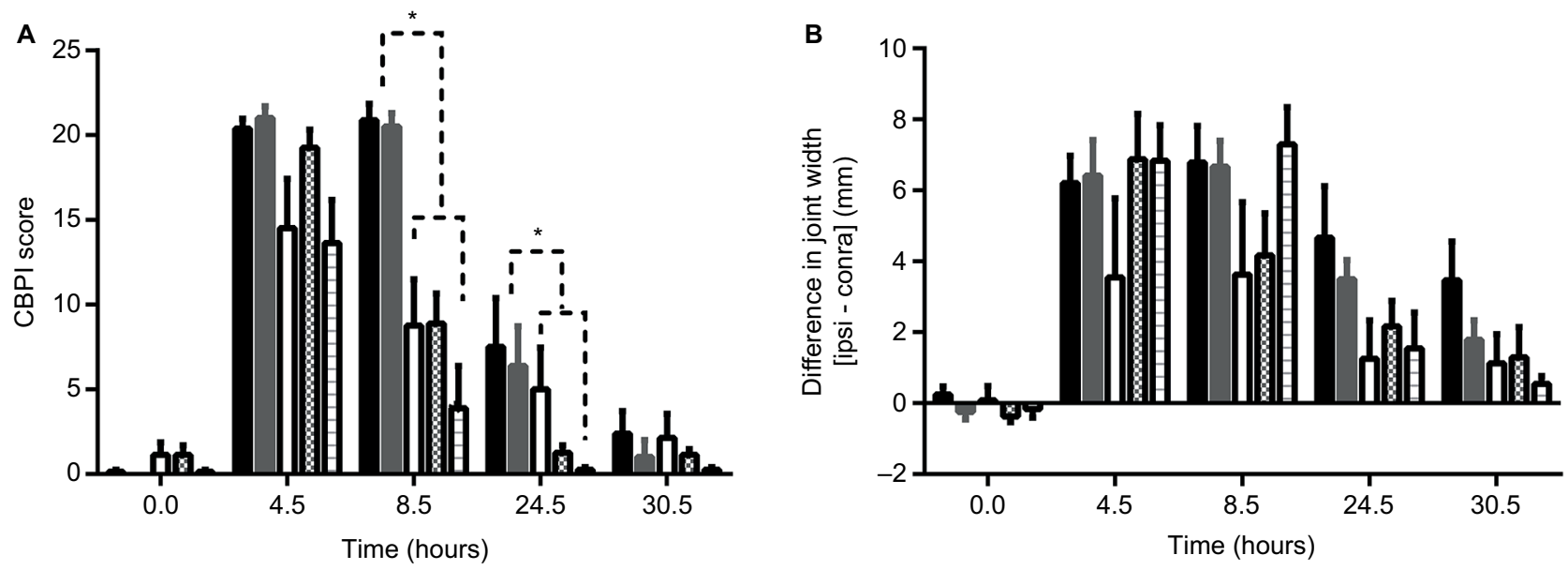

Figure $4 \mathrm{~A}$ comparison of oral and TD celecoxib to alter pain and swelling in a model of acute joint synovitis.

Notes: A study design involving beagle dogs ( $\mathrm{N}=8$ per group) involved baseline ( 0.0$)$ and post-synovitis measurements at $4.5,8.5,24.5$, and 30.5 hours for (A) pain levels using the CBPI and (B) swelling using stifle width $(\mathrm{mm})$ for the ipsilateral and contralateral joints. Inflammatory synovitis was induced by injection of $\mathrm{I} .0 \mathrm{~mL}$ of $20 \mathrm{mg} / \mathrm{mL}$ sodium urate (under anesthesia). Animals received no intervention (匹), vehicle Delivra ${ }^{\mathrm{TM}} \mathrm{SR}$ ( $100 \mathrm{mg} / \mathrm{kg}$;), or equimolar interventions consisting of oral celecoxib (3 mg/kg; ), PLO-ibuprofen ( $8 \%$ at $37.5 \mathrm{mg} / \mathrm{kg}$; ), or Delivra ${ }^{\mathrm{TM}}$-ibuprofen $(8 \%$ at $37.5 \mathrm{mg} / \mathrm{kg}$ ). Results are graphically represented as the average CBPI score and average joint width along with SEM error bars for each group. Linear mixed models for repeated measures and Bonferroni adjustment for multiple comparisons demonstrated all interventions yielding significant $(* P<0.02)$ and indistinguishable pain reduction $(\mathbf{A})$ at 8.5 and 24.5 hours.

Abbreviations: PLO, pluronic lecithin organogel; DEL, Delivra ${ }^{\mathrm{TM}}$; DEL-SR, Delivra ${ }^{\mathrm{TM}}$ slow release; CBPI, canine brief pain inventory; SEM, standard error of the mean; TD, transdermal; ipsi, ipsilateral; contra, contralateral.

$\mathrm{kg}$ /day) which resulted in $\mu \mathrm{g} / \mathrm{mL}$ levels in both serum and synovial fluid $(2,910 \mathrm{ng} / \mathrm{mL}$ and $1,817 \mathrm{ng} / \mathrm{mL}$, respectively, - Figure 1) one hour after the fourteenth administration. No comparable study of ibuprofen in canine synovial fluid could be identified; however, several human pharmacokinetic studies $\mathrm{s}^{31-35}$ have yielded $\mu \mathrm{g} / \mathrm{mL}$ concentrations in circulation and synovial fluid with at a ratio of $0.5-1.5$ for normal and arthritic individuals. ${ }^{31}$ In our study, we observe a similar level of total ibuprofen concentration as well as a serum to synovial fluid ratio consistent with this range (Figure 1). As such, DEL-SR can introduce ibuprofen to an extent similar to that of common oral dosages. The bioavailability of DEL-ibuprofen remains to be determined; however other topical formulations have yielded broad bio-availabilities 
of $0.5 \%-22 \%{ }^{36-38}$ Celecoxib levels in serum and synovial fluid ( $220 \mathrm{ng} / \mathrm{mL}$ and $203 \mathrm{ng} / \mathrm{mL}$ ) were demonstrably lower than for ibuprofen (Figures 1 and 2). Hunter et al reported peak synovial fluid celecoxib concentrations of $515 \mathrm{ng} /$ $\mathrm{mL}$ in greyhounds 1 hour after the fifth daily oral dose of approximately $11.8 \mathrm{mg} / \mathrm{kg}$ and plasma $\mathrm{C}_{\max }$ values of 3,907 $\mathrm{ng} / \mathrm{mL} .{ }^{39}$ Despite this, DEL-celecoxib yielded a similar serum to synovial fluid ratio of $\sim 1.0$ (Figure 2) to DEL-ibuprofen, suggesting that the mechanism of drug distribution may be equivalent across a broad spectrum of small pharmacological molecules. Whereas ibuprofen levels decreased significantly following a seven day washout period (Figure 1), celecoxib levels decreased but without achieving statistical significance (Figure 2). This suggests celecoxib may have a reduced clearance rate as compared to ibuprofen in canines. This initial penetration data supports the concept of TD NSAID application; however, a direct comparison between TD and oral with pharmacokinetics would provide a stronger understanding of bioequivalence and comparative bioavailability. Future studies will incorporate comparative pharmacokinetics during acute and chronic drug administrations for various routes.

The eligibility criteria employed in this animal model was consistent with those in prior clinical and pre-clinical studies that involved the induction of joint inflammation in test subjects; all prior studies included the confirmation that the test unit/subject have no prior history of osteoarthritis. Similar to the current study, Steele and McCarty used a sodium urate solution of $20 \mathrm{mg} / \mathrm{mL}$ of isotonic saline injected into the knees of male human volunteers with no history of arthritis. Symptoms of induced synovitis were observed within 1-2 hours and for some lasted for 8-10 hours post induction. ${ }^{40}$

Ibuprofen is known to result in significant GI issues in canines and is therefore not recommended for treating pain in dogs. ${ }^{14}$ This poses the question "how do you determine if the observation of pain and limitation in function in the oral ibuprofen group is a result of synovitis induction, rather than ibuprofen induced GI distress?" Symptoms of GI distress (nausea, vomiting, diarrhea, gastric ulceration, and abdominal pain) can be seen in doses of 50 to $125 \mathrm{mg} /$ $\mathrm{kg}^{14}$ and the dose administered in this study was far less. It should be noted that the animals were monitored for adverse events with no signs of GI distress reported. Also, the mean pain score for the PO ibuprofen group was $\sim 37 \%-38 \%$ of that reported for the untreated and vehicle control group at 8.5 hours (Figure 3 ). It is therefore unlikely that GI distress from ibuprofen ingestion was a confounder in this study.

In this animal model, interference with daily functions started at 4.5 hours and therapeutic amelioration was observed at 4.5, 8.5, and 24.5 hours (Figures 3 and 4). Administration of PO ibuprofen was the only intervention that showed a significant improvement in pain relief at 4.5 hours and all interventions significantly reduced pain at 8.5 and 24.5 hours, when compared to "no treatment." Due to the inevitable GI side effects associated with oral COX inhibitor use, adjunct therapy that combines a single oral and TD dose of a NSAID, extending pain relief up to 24 hours is a desirable and feasible alternative to current arthritis pain management options. This type of combination therapy should be further investigated in a human model. The advantage of the current model is that it permits rapid assessment that can be used to evaluate the time-course effects of an acute dose. The major limitation is that the pain is induced by sodium urate injection and reflects only acute pain. Confirmation of efficacy in a chronic model of gout, rheumatoid, or osteoarthritic pain models may offer further support for human clinical utility wherein NSAIDs are commonly used to treat chronic pain.

\section{Conclusion}

While several COX-2 selective NSAIDs have been tested and approved for use in dogs (e.g., deracoxib, mavacoxib, and firocoxib), the TD applicability of these drugs has not been investigated to date. To our knowledge this is the first evaluation of a COX-2 selective NSAID tested using a TD delivery platform for the alleviation of pain and edema in dogs. This warrants further development of TD NSAID formulations for treatment of arthritis in dogs. Topical vs oral NSAIDs have been assessed in numerous clinical studies in the treatment of arthritis and sports injuries. They frequently find analogous outcomes in patient pain and edema and topical treatments generate reduced instances of GI related side effects. The results of those studies and the current model support the assertion that DEL formulations of NSAIDs may have a comparable analgesic effect to that of oral administration of the same drug while limiting the negative off-target effect of active ingredients due to systemic exposure. These novel topical formulations of NSAIDs in DEL-SR should be further investigated as alternatives or as adjuncts to oral NSAID therapy in a controlled clinical experiment.

\section{Abbreviations}

$\mathrm{BLQ}$, below the limit of quantification; $\mathrm{C}_{\max }$, maximum concentration; CBPI, canine brief pain inventory; COX-1/2, cyclooxygenase enzymes 1/2; DEL, Delivra ${ }^{\mathrm{TM}}$; DEL-SR, Delivra $^{\mathrm{TM}}$ slow release; GI, gastrointestinal;; IS, Internal Standard; LC, liquid chromatography; LC-MS/MS, liquid chromatography tandem mass spectrometry; LLOQ, lower 
limit of quantification; MS, mass spectrometry; NSAID, nonsteroid ani-inflammatory drug; OTC, over the counter; PLO, pluronic lecithin organogel; PO, per oral; SEM, standard error of the mean; SPE, Solid phase extraction; TD, transdermal.

\section{Acknowledgments}

This project was sponsored by Delivra Corp. Formulation development of all test articles was performed by MNK Researchers Inc. We thank Professor Dr Henrik Stryhn of the Atlantic Veterinary College (Charlottetown, PE, Canada) in supporting the statistical analysis of data sets.

\section{Disclosure}

David C Baranowski, Beth Buchanan and Heather C Dwyer are all employees of Delivra Corp, the project sponsor. Joseph P Gabriele is the Chief Scientific Officer of Delivra Corp. The sponsor had no influence on the experiments, results, analysis of data, or writing of this manuscript. The author reports no other conflicts of interest in this work.

\section{References}

1. Zhang Y, Jordan JM. Epidemiology of osteoarthritis. Clin Geriatr Med. 2010;26(3):355-369.

2. McDougall C, Hurd K, Barnabe C. Systematic review of rheumatic disease epidemiology in the indigenous populations of Canada, the United States, Australia, and New Zealand. Semin Arthritis Rheum. 2017;46(5):675-686.

3. Teeple E, Jay GD, Elsaid KA, Fleming BC. Animal models of osteoarthritis: challenges of model selection and analysis. AAPSJ. 2013;15(2):438-446.

4. Malfait AM, Little CB, McDougall JJ. A commentary on modelling osteoarthritis pain in small animals. Osteoarthritis Cartilage. 2013;21(9):1316-1326.

5. Mayo Clinic Staff. Osteoarthritis: Symptoms, Causes, Diagnosis and Treatment. Mayo Clinic. Available from: https:/www.mayoclinic.org/ diseases-conditions/osteoarthritis/diagnosis-treatment/drc-20351930. Accessed OCtober 29, 2018.

6. Vane JR. Inhibition of prostaglandin synthesis as a mechanism of action for aspirin-like drugs. Nat New Biol. 1971;231(25):232-235.

7. Rainsford KD. Profile and mechanisms of gastrointestinal and other side effects of nonsteroidal anti-inflammatory drugs (NSAIDs). Am J Med. 1999;107(6A):27-35.

8. Whittle BJ. Gastrointestinal effects of nonsteroidal anti-inflammatory drugs. Fundam Clin Pharmacol. 2003;17(3):301-313.

9. Dunayer E. Ibuprofen toxicosis in dogs, cats, and ferrets. Vet Med. 2004:580-586. Available from: https://www.aspcapro.org/sites/default/ files/t-toxbrief_0704.pdf. Accessed May 23, 2018.

10. Innes $J$, O'Neill T, Lascelles D. Use of non-steroidal anti-inflammatory drugs for the treatment of canine osteoarthritis. In Pract. 2010;32(4):126-137.

11. Mason L, Moore RA, Edwards JE, Derry S, Mcquay HJ. Topical NSAIDs for chronic musculoskeletal pain: systematic review and meta-analysis. BMC Musculoskelet Disord. 2004;5(1):28.

12. Dominkus M, Nicolakis M, Kotz R, Wilkinson FE, Kaiser RR, Chlud $\mathrm{K}$. Comparison of tissue and plasma levels of ibuprofen after oral and topical administration. Arzneimittelforschung. 1996;46(12): 1138-1143.

13. Kuyinu EL, Narayanan G, Nair LS, Laurencin CT. Animal models of osteoarthritis: classification, update, and measurement of outcomes. $J$ Orthop Surg Res. 2016;11(1):1-27.
14. Richardson JA. Management of Acetaminophen and Ibuprofen Toxicoses in Dogs and Cats. Urbana, IL: DVMASPCA Animal Poison Control Center. The Journal of Veterinary Emergency and Critical Care.

15. Essex MN, Bhadra P, Sands GH. Efficacy and tolerability of celecoxib versus naproxen in patients with osteoarthritis of the knee: a randomized, double-blind, double-dummy trial. J Int Med Res. 2012;40(4):1357-1370.

16. Moore RA, Derry S, Makinson GT, McQuay HJ. Tolerability and adverse events in clinical trials of celecoxib in osteoarthritis and rheumatoid arthritis: systematic review and meta-analysis of information from company clinical trial reports. Arthritis Res Ther. 2005;7(3):R644-R665.

17. Cheung R, Krishnaswami S, Kowalski K. Analgesic efficacy of celecoxib in postoperative oral surgery pain: a single-dose, two-center, randomized, double-blind, active- and placebo-controlled study. Clin Ther. 2007;29(Suppl 11):2498-2510.

18. Nissen SE, Yeomans ND, Solomon DH, et al. Cardiovascular Safety of Celecoxib, Naproxen, or Ibuprofen for Arthritis. $N$ Engl J Med. 2016;375(26):2519-2529.

19. Tacconelli S, Capone ML, Sciulli MG, Ricciotti E, Patrignani P. The biochemical selectivity of novel COX-2 inhibitors in whole blood assays of COX-isozyme activity. Curr Med Res Opin. 2002;18(8):503-511.

20. Mukherjee D, Nissen SE, Topol EJ, Risk of cardiovascular events associated with selective COX-2 inhibitors. JAMA. 2001;286(8): 954-959.

21. Wadsworth LT, Kent JD, Holt RJ. Efficacy and safety of diclofenac sodium $2 \%$ topical solution for osteoarthritis of the knee: a randomized, double-blind, vehicle-controlled, 4 week study. Curr Med Res Opin. 2016;32(2):241-250.

22. Efe T, Sagnak E, Roessler PP, et al. Penetration of topical diclofenac sodium $4 \%$ spray gel into the synovial tissue and synovial fluid of the knee: a randomised clinical trial. Knee Surg Sports Traumatol Arthrosc. 2014;22(2):345-350.

23. Klinge SA, Sawyer GA. Effectiveness and safety of topical versus oral nonsteroidal anti-inflammatory drugs: a comprehensive review. Phys Sportsmed. 2013;41(2):64-74.

24. Vincent TL, Williams RO, Maciewicz R, Silman A, Garside P; Arthritis Research UK animal models working group. Mapping pathogenesis of arthritis through small animal models. Rheumatology. 2012;51(11):1931-1941.

25. Helmick CG, Felson DT, Lawrence RC, et al. Estimates of the prevalence of arthritis and other rheumatic conditions in the United States. Part I. Arthritis Rheum. 2008;58(1):15-25.

26. Lawrence RC, Felson DT, Helmick CG, et al. Estimates of the prevalence of arthritis and other rheumatic conditions in the United States. Part II. Arthritis Rheum. 2008;58(1):26-35.

27. Martel-Pelletier J, Barr AJ, Cicuttini FM, et al. Osteoarthritis. Nat Rev Dis Primers. 2016;2:16072.

28. Smolen JS, Aletaha D, Barton A, et al. Rheumatoid arthritis. Nat Rev Dis Primers. 2018;4:18001.

29. So AK, Martinon F. Inflammation in gout: mechanisms and therapeutic targets. Nat Rev Rheumatol. 2017;13(11):639-647.

30. Borer LR, Seewald W, Peel JE, King JN. Evaluation of the dose-response relationship of oral robenacoxib in urate crystal-induced acute stifle synovitis in dogs. J Vet Pharmacol Ther. 2017;40(2):148-157.

31. Rainsford KD. Ibuprofen: pharmacology, efficacy and safety. Inflammopharmacology. 2009;17(6):275-342.

32. Gallo JM, Gall EP, Gillespie WR, Albert KS, Perrier D. Ibuprofen kinetics in plasma and synovial fluid of arthritic patients. J Clin Pharmacol. 1986;26(1):65-70.

33. Mäkelä AL, Lempiäinen M, Ylijoki H. Ibuprofen levels in serum and synovial fluid. Scand J Rheumatol Suppl. 1981;39:15-17.

34. Glass RC, Swannell AJ. Concentrations of ibuprofen in serum and synovial fluid from patients with arthritis [proceedings]. $\mathrm{Br} \mathrm{J}$ Clin Pharmacol. 1978;6(5):P453-P454. 
35. Cox SR, Gall EP, Forbes KK, Gresham M, Goris G. Pharmacokinetics of the $\mathrm{R}(-)$ and $\mathrm{S}(+)$ enantiomers of ibuprofen in the serum and synovial fluid of arthritis patients. J Clin Pharmacol. 1991;31(1):88-94.

36. Tegeder I, Muth-Selbach U, Lötsch J, et al. Application of microdialysis for the determination of muscle and subcutaneous tissue concentrations after oral and topical ibuprofen administration. Clin Pharmacol Ther. 1999;65(4):357-368.

37. Seth PL. Percutaneous absorption of ibuprofen from different formulations. Comparative study with gel, hydrophilic ointment and emulsion cream. Arzneimittelforschung. 1993;43(8):919-921.
38. Kleinbloesem CH, Ouwerkerk M, Spitznagel W, Wilkinson FE, Kaiser RR. Pharmacokinetics and bioavailability of percutaneous ibuprofen. Arzneimittelforschung. 1995;45(10):1117-1121.

39. Hunter RP, Radlinsky M, Koch DE, Corse M, Pellerin MA, Halstead J. Plasma pharmacokinetics and synovial fluid concentrations after oral administration of single and multiple doses of celecoxib in greyhounds. Am J Vet Res. 2005;66(8):1441-1445.

40. Steele AD, McCarty DJ. An experimental model of acute inflammation in man. Arthritis Rheum. 1966;9(3):430-442.

\section{Publish your work in this journal}

The Journal of Pain Research is an international, peer reviewed, open access, online journal that welcomes laboratory and clinical findings in the fields of pain research and the prevention and management of pain. Original research, reviews, symposium reports, hypothesis formation and commentaries are all considered for publication.
The manuscript management system is completely online and includes a very quick and fair peer-review system, which is all easy to use. Visit http://www.dovepress.com/testimonials.php to read real quotes from published authors. 